\title{
Correction to: Establishment of Neurobehavioral Assessment System in Tree Shrew SCT Model
}

\author{
Yang-Yang Wang ${ }^{1,3} \cdot$ Jie -Dong Wang ${ }^{2} \cdot$ Lei Wang $^{5} \cdot$ Qi-Qin Dan ${ }^{3} \cdot$ Qing- Jie Xia ${ }^{3} \cdot$ Ting-Hua Wang $^{2,3}$. \\ Liu-Lin Xiong ${ }^{1,3,4}$
}

Published online: 9 November 2020

○) Springer Science+Business Media, LLC, part of Springer Nature 2020

\section{Correction to: Journal of Molecular Neuroscience (2020) 70:308-319. \\ https://doi.org/10.1007/s12031-019-01414-9}

The original version of this article unfortunately contained a mistake in the Funding section. The correct funding information is presented below:

Funding This study was supported by Research Fund for the Doctoral Program of Liu-Liu Xiong (201903), the Program Innovative Research Team in Science and Technology in Yunnan Province (No. 2017HC007), and the grant from the National Natural Science Foundation of China (Grant No. 81471268 and 81960214). We also would like to thank Professor Fei Liu of Department of Anesthesiology, West China Hospital, Sichuan University and Professor Zhao-Qiong Zhu of Department of Anesthesiology, The Affiliated Hospital of Zunyi Medical University for their technical support.

The original article can be found online at https://doi.org/10.1007/ s12031-019-01414-9.

Ting-Hua Wang

tinghua_neuron@263.net

$\triangle$ Liu-Lin Xiong

499465010@qq.com

1 Department of Anesthesiology, The Affiliated Hospital of Zunyi Medical University, Zunyi, Guizhou 563000, China

2 Institute of Neuroscience, Animal Zoology Department, Kunming Medical University, Kunming 650031, China

3 Institute of Neurological Disease, Translational Neuroscience Center, West China Hospital, Sichuan University, Chengdu 610041, China

4 School of Pharmacy and Medical Sciences, Sansom Institute, University of South Australia, Adelaide, South Australia 5000, Australia

5 Molecular Imaging Laboratory, Department of Radiology, West China Hospital, Sichuan University, Chengdu 610041, China
Publisher's Note Springer Nature remains neutral with regard to jurisdictional claims in published maps and institutional affiliations. 